\title{
Open versus Endovascular Abdominal Aortic Aneurysm Repair in VA Hospitals
}

\author{
by \\ Ruth L. Bush, MD \\ Assistant Professor of Medicine \\ Houston Center for Quality of Care and Utilization Studies \\ Michael E. DeBakey Veterans Affairs Medical Center \\ Baylor College of Medicine \\ Houston, TX
}

A Master's Paper submitted to the faculty of the University of North Carolina at Chapel Hill in partial fulfillment of the requirements for the degree of Master of Public Health in the Public Health Leadership Program.

Chapel Hill, NC

2005

Advisor:

Second reader:

Date: 


\section{Abstract}

Background: Endovascular abdominal aortic aneurysm repair (EVAR), when compared to conventional open surgical repair, has been shown to reduce perioperative morbidity and mortality. A retrospective cohort study was performed with data from the Department of Veterans Affairs to examine outcomes in routine daily practice following EVAR and open surgical repair. Methods: In this study, I examined 30-day mortality, 1-year survival, and postoperative complications in 1957 patients who underwent elective AAA repair (EVAR $n=717(36.6 \%)$; open $n=1240(63.4 \%)$ ) at 123 VA hospitals between May 1, 2001 and September 30, 2003. Also, this review investigated the influence of patient, operative, and hospital variables on outcome, and used propensity scoring to adjust for the nonrandom allocation of patients to either EVAR or open procedures.

Results: Patients undergoing EVAR had significantly lower 30 day (3.1\% vs. $5.7 \%, \mathrm{p}=0.0079)$ and 1 year mortality $(8.4 \%$ vs. $12.0 \%, \mathrm{p}=0.0121)$ than patients having open repair. EVAR was positively and independently associated with a decrease in 30-day postoperative mortality (adjusted $\mathrm{OR}=0.58 ; 95 \% \mathrm{CI}=0.35$, $0.97 ; p=0.0367)$. The risk of any perioperative complication was much less following EVAR (4.5\% vs. $14.2 \%$; $<<0.0001$; unadjusted OR $0.48 ; 95 \% \mathrm{CI}$ $=0.37,0.63 ; \mathrm{p}<0.001)$. Patients operated on at low volume hospitals $(25 \%$ of entire cohort) were more likely to have had open repair $(31.6 \%$ compared to $15.9 \%$ EVAR; $\mathrm{p}<0.001$ ) as well as a two-fold increased adjusted 30 -day mortality risk $(\mathrm{OR}=2.06 ; 95 \% \mathrm{CI}=1.32,3.26 ; \mathrm{p}=0.0015)$. 
Conclusions: In routine daily practice, patients presenting for elective AAA repair who undergo EVAR have substantially lower perioperative mortality and morbidity rates compared to patients having open repair. The benefits of a minimally invasive approach were readily apparent in this cohort; however, caution should be exercised in choosing EVAR for all elective AAA repairs until longer-term data on device durability are available. Furthermore, widely available screening programs may aid in the early detection of AAA, which would further facilitate timely surgical intervention and, ultimately, improve outcomes. 


\section{Introduction}

The goal of elective repair of abdominal aortic aneurysms (AAA) is to improve longevity by preventing aneurysm rupture and subsequent death. The introduction of endovascular grafting, the treatment of an AAA by inserting an endovascular graft retrograde through an open femoral artery into the abdominal aorta, by Juan Parodi in 1991, was a milestone in the management of patients with AAA. This technical advance has been embraced with enthusiasm because of the potential for reducing perioperative morbidity and mortality, and recovery time, especially in patients deemed at high surgical risk by conventional open standards. ${ }^{2}$ However, defining an appropriate strategy for choosing either conventional open or endovascular repair remains a complex clinical endeavor. Even minimally invasive interventions may be associated with adverse early outcomes, and the presumption that an endovascular approach confers less risk for perioperative mortality compared with the results of standard open surgery in patients at low risk remains unproven.

Single institution series or clinical experiences from controlled industrysponsored trials have populated the literature, with illustrative data showing a positive impact on mortality rate following intact infrarenal AAA repair. ${ }^{3-7}$ In a meta-analysis published in 2002 , the results of 9 studies involving 1,318 procedures (687 EVAR and 631 open surgical repair) was reported, with an aggregate 30 -day mortality rate of $3 \%$ for EVAR and $4 \%$ for open repair $(p=0.03)^{8}$ Additionally, in 2004, short-term data (30-day) from the first 2 
randomized trials comparing elective EVAR to open repair have demonstrated superiority of EVAR in reducing perioperative mortality. 9,10

The main purpose of this study was to compare postoperative mortality and complications and 1 year survival in patients undergoing elective EVAR versus open repair within a national publicly funded health care system. Secondarily, I will discuss the importance of screening programs to improve and expedite the diagnosis, and ultimately, quality of delivered care to patients with AAAs.

\section{Methods}

\section{Data Sources and Sample}

All patients who underwent either EVAR or open abdominal aortic aneurysm repair between May 1, 2001 and September 30, 2003 were identified through the Veterans Health Administration's (VA) National Surgical Quality Improvement Program (NSQIP) database ${ }^{11}$ of surgical procedures performed at 123 participating VA hospitals. This time frame was chosen because prior to May 2001, no suitable current procedural terminology (CPT) codes existed in the database for EVAR, though endovascular grafting systems were approved by the US Food and Drug Administration and became commercially available in September 1999. Patients undergoing elective repair were defined by primary diagnostic code (ICD-9-CM 441.4: intact, non-ruptured AAA); open AAA repairs were defined by CPT codes $35081,34830,34831$, and 34832 . The last 3 codes represent elective open repair after EVAR; thus, a non-urgent operation with 
similar risks of primary open repair (data analysis was not performed in an intentto-treat manner). EVAR was defined by codes 34800,34802 , and 34804. Patients with secondary diagnostic codes for ruptured AAA or thoracic or thoracoabdominal aortic aneurysm were excluded from the analysis.

The NSQIP database contains very detailed, prospectively collected clinical data on all patients undergoing major surgical procedures within the VA. ${ }^{11}$ At the time of surgery, patients are enrolled in NSQIP, and baseline demographic, pre-operative laboratory and clinical information is collected by dedicated trained nurse reviewers. Additional perioperative data are prospectively collected by the nurses, including 30-day morbidity and mortality information. From the NSQIP database we identified 1973 elective repairs of non-ruptured aneurysms, which occurred between May 2001 and September 2003. Of these, 9 records were found to represent a second operation on the same individual; these 9 records were excluded from analysis.

To supplement the information in the NSQIP records with longer-term utilization and vital statistics data, encrypted social security numbers, date of admission, date of discharge, and primary operative date were used to link the NSQIP database with the VA Patient Treatment File (PTF), which contains abstracts of all patients discharged from all VA hospitals. Seven patients $(0.36 \%)$ for whom a match could not be made were excluded from further study. Thus, 1957 unique patients were included in for analysis. A total of 717 EVAR and 1240 open cases formed the basis of this study. Of the 1240 open cases, $53(4.3 \%)$ patients had elective open repairs following EVAR. To obtain additional 
information, linkages were performed with the VA Outpatient Clinic File (OPC), which contains records for every outpatient visit to a VA facility, and the VA Beneficiary Identification Record Locator System (BIRLS) death file. Approval for the study was obtained from the Baylor College of Medicine Institutional Review Board and the Michael E. DeBakey VA Medical Center Research and Development Review Committee as well as the University of North Carolina School of Public Health Institutional Review Board.

Definition of independent variables

Patient demographic data are recorded prospectively in the NSQIP database including age, gender, race, and hospital location. Comorbid conditions known to have an influence on the risk of cardiovascular morbidity and mortality were chosen for analysis. ${ }^{2,12,13}$ These independent variables were defined using NSQIP definitions and the International classification of diseases, ninth revision, Clinical modification (ICD-9-CM) codes. Within NSQIP, nurse reviewers recorded diabetes (persons using insulin or oral hypoglycemic agent), renal dysfunction or dialysis dependence, hepatic dysfunction, history of malignancy, history of congestive heart failure during the month preceding surgery, history of stoke or transient ischemic attacks, functional status, and tobacco use within the year preceding AAA repair. Additionally, 24 individual comorbid conditions were identified in the PTF and the analysis also included a separate variable for elevated creatinine ( $\geq 2.0 \mathrm{mg} / \mathrm{dL}$ ) which was based on the Revised Cardiac Risk Index. ${ }^{13}$ American Society of Anesthesiology (ASA) classification. Both the 
inpatient and outpatient files were searched for matching ICD-9-CM codes 1 year prior to the operation date in order to have complete patient comorbidity profiles. Baseline characteristics of patients are given in Table 1. EVAR patients consisted of a significantly older population with progressively increasing percentages of patients having EVAR compared to open repair in the older age categories. Younger patients were more likely to have open repair. Patients undergoing EVAR were significantly less likely than patients having open repair to have a history of cerebrovascular disease, to have smoked within the year prior to AAA repair, to have a carotid lesion, and to have been operated on at low volume hospitals and more likely to have had cirrhosis. Overall, the two cohorts were remarkably similar given the retrospective nature of the analysis.

\section{Outcome Measures}

The outcomes of interest included 30-day and 1 year mortality, and any postoperative complications. Thirty-day mortality was obtained from the NSQIP database; 1-year mortality was calculated using death dates obtained from BIRLS and the PTF. Perioperative complications from the NSQIP were aggregated into categories including adverse cardiac events, renal dysfunction, pulmonary complications, wound complications, neurological complications, and graft failure. Unfortunately, there is no strict definition in the NSQIP for what constitutes a "graft failure", thus, this variable may be misleading and difficult to make conclusions from. Postoperative length of stay and ICU length of stay were calculated using the date of surgical repair as the index date. 
While a direct correlation between high volume hospitals and better outcomes for open elective AAA repair has been well documented in the literature, no study has assessed the significance of the volume effect for endovascular repair. ${ }^{14-16}$ Because of potential confounding due to the volume of operations performed at each site, we created an indicator variable for low volume, for all patients who had their surgery at a hospital which performed fewer than 10 procedures (EVAR or open) per year. ${ }^{15}$ Patients operated on from May 2001 through September 2001 were classified as being at low volume sites if those sites performed fewer than 4 operations during this abbreviated time period. Statistical Analysis

Bivariate tests of association with type of surgery were performed for all demographic and clinical characteristics by the use of chi-square tests for categorical variables and t-tests for continuous variables (age). All clinical outcomes of interest were similarly tested for association with type of surgery performed. All independent measures and type of surgery were then tested for their unique association with the morbidity and mortality outcomes (30-day and 365-day) in multivariable logistic regression models with a significance level of 0.10 required to stay in the final model. This level was selected arbitrarily to capture as many possible confounding factors as might be strongly associated with both the selection of EVAR and postoperative outcomes. Age was tested for linear association and found to have the best empirical fit as a categorical variable (greater than or equal to 80 years). An interaction term of low volume with type of surgery was not statistically significant. Models were assessed for goodness of 
fit by the Hosmer-Lemeshow statistic and for discrimination by the c-index. ${ }^{17-19}$ Further testing of time to death was done with Kaplan-Meier curves evaluated with the log-rank test.

Propensity scoring was used to try to minimize the selection bias inherent in this nonrandomized observational study. The predicted probability of receiving EVAR was tabulated for all patients, based on a multiple logistic regression of all independent demographic and clinical characteristics, and stratified into 5 groups. Patients were thus matched within strata based on similar levels of the independent measures, creating a pseudo-randomized control design. ${ }^{20}$ The proportion of patients with the morbidity and mortality outcomes was then compared between the EVAR and open procedures between strata.

All analyses were performed using SAS Unix version 9.0. All other significance tests were conducted at an alpha level $=0.05$.

\section{Results}

Patients undergoing EVAR had statistically significantly better outcomeslower short and long-term mortality rates, lower complication rates, and shorter hospital and ICU length of stays- than patients undergoing traditional open aneurysm repair (Table 2 ).

\section{Mortality}

Patients undergoing EVAR had a lower risk of postoperative mortality than those having open repair (Table 3 ). The adjusted odds ratio (95\% CI and pvalue) of EVAR for 30-day mortality, after controlling for statistically significant 
independent risk factors, was $0.58(0.35,0.97 ; \mathrm{p}=0.0367)$. One-year survival was also significantly better in the EVAR cohort with an adjusted OR of $0.59(0.42$, $0.84 ; p=0.0028)$. Patients operated on at low volume hospitals, regardless of surgery type, had a more than two-fold increase in risk $(\mathrm{OR}=2.06, \mathrm{CI} 1.32,2.30$, $\mathrm{p}=0.0015$ ) for 30 -day mortality. Patients at low volume sites were also at increased risk for one-year mortality, albeit not statistically significant (OR 1.35, CI $0.97,1.88, \mathrm{p}=0.078$ ). Kaplan-Meier analysis (Figures 1.a and 1.b) further demonstrated significant differences in both 30 -day $(\mathrm{p}=0.0058)$ and 1 year $(p=0.0104)$ survival rates. Furthermore, after stratifying the patients by their propensity to undergo EVAR or open surgery, the proportion of patients with deaths within one year were higher among patients receiving open repair in all groups substantiating our results (Figure 2).

Postoperative complications

Patients undergoing EVAR had a significantly lower overall adjusted rate of postoperative complications (OR $0.48 ; 95 \%$ CI $0.37,0.63 ; \mathrm{p}<0.0001$ ). In bivariate analysis, cardiac and pulmonary complications as well as graft failure reached statistical significance (Table 4). There were 3 instances of complete graft failure in which the endovascular graft was removed and traditional open repair completed. Two of these cases occurred within 30 days of EVAR and one case was 5 months post-implantation. After controlling for type of surgery, independent predictors for an increased risk of mortality and complications in risk-adjusted models were hepatic comorbidity, cancer diagnosis, arrhythmias, renal insufficiency, ASA class 4 or 5, transient ischemic attack, history of COPD, 
history of $\mathrm{CHF}$, hypertensive renal function, cirrhosis, angina, and carotid disease. There was no difference in complication rates between lower and higher volume hospitals $(\mathrm{p}=0.16)$.

Length of stay

Patients having EVAR utilized significantly fewer hospital days than those having open repair. The median ICU length of stay for EVAR was 1 day (interquartile range 1,2 days) and for open repair was 4 days (interquartile range 2, 6 days). Total postoperative length of stay was also shorter following EVAR than open repair (EVAR: median 3, interquartile range 2, 5 days; open: median 7 , interquartile range 6,11 days).

Propensity score analysis

Figure 2 presents the outcomes of patients matched on propensity to undergo EVAR surgery, stratified by the probability of undergoing EVAR vs. open repair. Patients in the middle group were equally likely to have open or EVAR repair, based on their presenting demographic and clinical characteristics. Patients in the left-most group on the chart were most likely to have open, whereas patients in the right-most group were most likely to have EVAR. The proportion of patients with 30 -day death was higher for those patients having open repair, in all sub-groups except moderate-probability EVAR. The proportion of patients with deaths within one year were higher among patients receiving open repair in all groups, and the proportion of patients with occurrence of any complication were higher among patients receiving open repair in all groups except those equally likely to have either operation. The proportions of patients 
experiencing these morbidity and mortality outcomes generally decreased as the probability of EVAR increased. Conversely, the proportion of patients operated on at low volume hospitals increased dramatically as the probability of open repair increased $(88.2 \%)$, where patients also had the highest proportions of these adverse outcomes.

\section{Discussion}

In this cohort of patients, representing routine surgical practice, patients presenting for elective AAA repair who underwent EVAR had statistically significantly lower risk-adjusted perioperative and 1-year mortality, lower complication rates, and shorter lengths of stay compared to patients having open repair. The sample was restricted by querying the database for only those cases performed after the introduction of endovascular CPT codes in May 2001. These findings with regard to outcomes following EVAR and open AAA repair are consistent with several other published studies including those using national administrative databases, with additional 1-year survival via database linkage.

All of the perioperative outcomes analyzed, including 30 day and 1-year mortality rates, postoperative complications, and length of stay, favored endovascular repair in this veteran population. Patients undergoing open AAA repair were found to be younger and had a higher prevalence of cerebrovascular disease and carotid lesions, and lower prevalence of cirrhosis than those undergoing EVAR. Open patients were also more likely to have smoked within the year preceding repair. Otherwise, the 2 cohorts were well matched in terms of 
preoperative comorbid conditions including ischemic heart disease, history of myocardial infarction, pulmonary and renal function, all of which are known to influence postoperative outcomes after elective AAA repair. ${ }^{21}$

There are several competing explanations for the results of our analyses. The foremost reason is that EVAR is simply a superior treatment modality for elective aortic aneurysm repair. Studies have been performed measuring changes in physiologic response via plasma catecholamine concentrations, changes in cardiovascular variables, and acid-base status in patients undergoing either elective EVAR or open repair. ${ }^{22-24}$ The less invasive endoluminal operation results in a lowering of the hypermetabolic stress response. Additionally, significant changes have been documented in various cardiovascular parameters including cardiac output, mean arterial pressure, and systemic vascular resistance during open repair related to aortic cross-clamping. ${ }^{24}$ Acute aortic occlusion (cross-clamping) and the subsequent reperfusion of the lower extremities are necessary maneuvers during open repair that may result in varying degrees of hemodynamic instability in a patient population intrinsically at high risk for cardiovascular disease.

An additional explanation for our findings is selection bias for patients to have either type of repair depending on the treating facility and the access to endovascular repair. EVAR is a technically demanding procedure not extensively taught in vascular surgical training programs until recent years, so it may not be offered at every hospital. Our propensity scoring demonstrated that EVAR tended 
to have lower 30-day and 1-year mortality compared to open repair in almost all propensity score subgroups.

In subset analysis, it was found that $25 \%$ of all patients had been treated at low volume hospitals (those performing $<10$ AAA repairs per year) and $41 \%$ of these patients died within 30 days of their operation. It has been suggested that veteran patients may have more comorbidities and a greater severity of illness than non-VA sector patients ${ }^{25,26}$, thus, one may expect worse results following specific surgical procedures such as open AAA repair, especially in lower volume institutions. Therefore, the non-VA population may have even better outcomes than seen in this NSQIP study. Specific reasons for this are not available in this dataset. On the other hand, there is evidence for better outcomes following elective open repair of smaller aneurysms in fit patients. ${ }^{27,28}$ However, aneurysm size is not currently recorded in the existing VA database.

Many believe the question of which operation to perform for elective AAA repair would best be answered by a prospective multicenter randomized trial. Such a trial (VA Cooperative Trial \#498) is currently underway in the VA health system and will span 5-7 years involving 39 centers. However, the results of recent randomized trials of EVAR versus open repair demonstrate a 30-day mortality advantage for EVAR patients; long term data are not yet available. ${ }^{9,10} \mathrm{~A}$ factor in interpreting results of the recently published trials, is that to be considered a candidate for randomization, a patient has to be eligible for either operation. The suitability for EVAR is reported to range from only $20 \%$ to $60 \%$ depending on anatomical criteria or device availability. ${ }^{29-31}$ This data set used 
herein may represent more accurately the spectrum of medical care than the setting of a randomized experiment. Furthermore, generalizability of the results of a prospective randomized trial to everyday common surgical practice may not be possible.

Using this prospectively collected observational data combined with administrative data, a propensity score approach was employed to replicate randomization. By using this statistical modality that grouped patients with similar risk profiles on the basis of their propensity to undergo either open AAA repair or EVAR, a comparison was performed for 30-day operative mortality between those who actually had open AAA repair or EVAR. After the patients were matched by propensity to undergo a certain type of operation, differences between the two groups became apparent, with higher mortality rates in those having had open AAA repair irrespective of the propensity score to undergo open repair. These findings differ from others and are more reliable in that all confounding background variables have been reduced into one single composite characteristic. By performing subclassification, simultaneously adjustment for many covariates is accomplished which allows for a reliable estimation of the effects of open repair versus EVAR. Other large-scale database analyses have relied on standard regression-based risk adjustment techniques, which are limited in their inability to assure balance in the distributions of covariates between types of operation performed.

Multiple authors have discussed the relationship between hospital volume and risk-adjusted outcome for AAA repair. ${ }^{14,16,32,33}$ Varying definitions for what 
constitutes a low-volume center have been suggested based on post-hoc analyses. A recent systematic review of 15 studies published from 1980 to 1997 on outcome related to hospital volume reported the number of median cases per year defining a low volume hospital as 10 (range 5 to 50 ). ${ }^{15}$ To assess whether these findings concerning lower volume centers may have biased our mortality findings in favor of higher volume hospitals, the adjusted one-year mortality rate was determined in separate multivariate models for patients from low volume versus high volume hospitals (data not shown). The results were consistent, and furthermore, the models demonstrated crossover effects of survival. One year after AAA repair, survival was higher in the very low volume hospitals compared to high volume hospitals. This finding suggests that though the sicker, more highrisk patients who are treated at high volume hospitals may survive the initial operation, most likely they are succumbing to pre-existing conditions such as heart disease. Alternatively, this finding proposes that differences in outcomes by hospital volume are not necessarily attributable to variations in patient characteristics or perioperative complication rates. The healthier counterparts who are repaired at lower volume hospitals and endure the primary operation have improved 1-year survival. These possible explanations of this data are consistent with others' findings in that though initial survival rates are higher with EVAR compared to open repair, any operative advantage disappears by 1-year following the operation. ${ }^{5,21}$

The results presented herein impart several unique contributions to the current body of knowledge. These data were obtained from a nationwide 
database, which is very detailed and prospectively collected by uniformly trained personnel. NSQIP includes 123 institutions with undoubtedly more than 123 surgeons and surgical teams performing elective AAA repairs. Thus, this data set is robust with respect to variability in surgical techniques and skills as well as in cohort size. Furthermore, NSQIP does not have the patient exclusion criteria that randomized trials contain, increasing the generalizability of our results.

The results of the 2 recent prospective multicenter randomized trials demonstrated that when cohorts were matched for comorbid conditions, the 30day operative mortality was reduced by treatment by EVAR. ${ }^{9,10}$ The results presented in this manuscript reflect these findings, albeit in an observational design, giving more confidence to the superiority of EVAR in the short-term and at one year. However, both of the prospective studies, many retrospective reviews, and this study caution practitioners about changes in clinical practice until further information on graft durability and failure rates is known. Currently available devices have experienced device migration, endoleak, and limb thrombosis, resulting in a high rate of secondary procedures and, in rare instances, failure to safeguard the patient from aneurysm rupture. Within the NSQIP database, reporting of endovascular device failures is not specifically documented, thus, the true rate of graft failure cannot be determined.

Of late, the subject of screening for AAA has come to the forefront for policy makers. Similar to colonoscopy and mammography, screening for AAA would benefit those individuals who may be at most risk for having an AAA and those who may benefit from surgical intervention. Screening ultrasonography for 
AAA in asymptomatic persons is a highly accurate test, with $95 \%$ sensitivity and near $100 \%$ specificity. ${ }^{34,35}$ In the $108^{\text {th }}$ Congress, a bill was introduced on June 21 , 2004 by Greenwood (Rep: Texas). This bill recommends amending title XVIII of the Social Security Act to provide coverage of ultrasound screening for AAA under Medicare part B. This bill was called the "SCAVE" bill which stands for "screen abdominal aortic aneurysms very efficiently" and was supported by the National Aneurysm Alliance.

Controversy has surrounded the issue of population-based AAA screening. Advocates state that ultrasound may detect AAAs at a size when rupture is unlikely to occur, and therefore, provide an opportunity for early intervention. In a 2001 study, over 70,000 men age 65 years and over were recruited to participate in a screening program within the United Kingdom (Multicentre Aneurysm Screening Study). ${ }^{36}$ Patients were randomized to receive either screening or routine care (no screening) and followed for 4 years. The results found a $53 \%$ reduction (95\% CI 30-64) in those who had screening mainly due to early intervention, thus, suggesting beneficial outcomes from a screening program. Conversely, a similar trial from Western Australia $(n=41,000)$ did not find a survival benefit with the exception of selected subpopulations of older men. ${ }^{37}$ These data, along with other compelling data from randomized clinical trials comparing screened versus nonscreened populations, were considered in policy establishment. ${ }^{35,38,39}$ With influence from national organizations including the Society of Vascular Surgery and the Society for Vascular Medicine and Biology, 
the U.S. Preventative Services Task Force published clinical guidelines for AAA screening this year. ${ }^{40}$ These guidelines are listed in Table 5.

There are several limitations to this study. This review did not have aneurysm size data within the databases analyzed. If surgeons began using EVAR on healthier patients with small aneurysms, which would not have otherwise been repaired, then the results of EVAR may appear better. With propensity scoring, simulating pseudo-randomization randomization, selection bias is decreased, although not eliminated. Additionally, use of the BIRLS database, which contains veteran death dates regardless whether they occur in a VA facility or elsewhere, has been demonstrated to miss approximately $5 \%$ to $10 \%$ of veteran decedents. ${ }^{29}$ However, there is no reason to believe that more EVAR deaths than open deaths were overlooked. The use of hospital volume as a surrogate marker for individual surgeon caseload or outcomes may not be representative of individual results. No current consensus exists on the minimum number of procedures needed to maintain or achieve appropriate outcome, however, it is intuitive that higher volume centers with more experienced surgeons performing procedures on a regular basis will achieve better results.

The results from this retrospective analysis open several avenues for future research endeavors which I plan to explore via potential national/federal funding mechanisms. With this pilot data, numerous questions of interest have been raised which will be addressed in a larger project grant (VA Investigator Initiated Research grant) to be submitted late 2005. My plans include updating the database to increase the statistical power and be able to perform subset analyses 
(age differences, racial disparities, geographical treatment variation, and further hospital volume analyses). Additionally, I will explore data collection options to gather AAA size data from all of the hospitals participating in the NSQIP database as well as information on long-term graft failure or secondary interventions. By continuing this area of research and data analysis, I would like to contribute to the information practitioners have at hand for both optimum patient guidance and treatment decision-making.

\section{Conclusion}

In this large patient cohort, representing routine clinical practice, endovascular repair had superior outcomes to open repair. Patients undergoing EVAR had better survival rates and shorter hospital stays than patients having open repair. A cautionary note remains regarding long-term graft durability as endovascular technology is still in a state of evolution. The institution of widely available population-based screening programs may detect asymptomatic aneurysms at a smaller size in healthier patients, which are amenable either to elective repair or observation. 


\section{References}

1. Parodi J, Palmaz J, Barone H. Transfemoral intraluminal graft implantation for abdominal aortic aneurysm. Ann Vasc Sueg 1991;5:491-499.

2. Buth J, van Marrewijk CJ, Harris PL, Hop WC, Riambau V, Laheij RJ. Outcome of endovascular abdominal aortic aneurysm repair in patients with conditions considered unfit for an open procedure: a report on the EUROSTAR experience. $J$ Vasc Surg 2002;35(2):211-21.

3. Zarins C, White R, Moll F, et al. The AneuRx stent graft: Four-year results and worldwide experience 2000. J Vasc Surg 2001;33:S135-145.

4. Zarins C, White R, Schwarten D, et al. AneuRx stent graft versus open surgical repair of abdominal aortic aneurysms: multicenter prospective clinical trial. J Vasc Surg 1999;29:292-308.

5. Moore W, Kashyap V, Vescera C, Quinones-Baldrich W. Abdominal aortic aneurysm: a six-year comparison of endovascular versus transabdominal repair. Annals of Surgery 1999;230:298-305.

6. May J, White $\mathrm{G}, \mathrm{Yu} \mathrm{W}$, et al. Concurrent comparison of endoluminal versus open repair in the treatment of abdominal aortic aneurysms: analysis of 303 patients by life table method. J Vasc Surg 1998;27:213-221.

7. Arko FR, Hill BB, Olcott C, Harris EJ, Fogarty TJ, Zarins CK. Endovascular repair reduces early and late morbidity compared to open surgery for abdominal aortic aneurysm. $J$ Endovasc Ther 2002;9(6):711-8.

8. Adriaensen ME, Bosch JL, Halpern EF, Myriam Hunink MG, Gazelle GS. Elective endovascular versus open surgical repair of abdominal aortic aneurysms: systematic review of short-term results. Radiology 2002;224(3):739-47.

9. Greenhalgh RM, Brown LC, Kwong GP, Powell JT, Thompson SG. Comparison of endovascular aneurysm repair with open repair in patients with abdominal aortic aneurysm (EVAR trial 1), 30-day operative mortality results: randomised controlled trial. Lancet 2004;364(9437):8438.

10. Prinssen $M$, Verhoeven EL, Buth J, et al. A randomized trial comparing conventional and endovascular repair of abdominal aortic aneurysms. $N$ Engl J Med 2004;351(16):1607-18.

11. Khuri SF, Daley J, Henderson W, et al. The Department of Veterans Affairs' NSQIP: the first national, validated, outcome-based, risk-adjusted, and peer-controlled program for the measurement and enhancement of the quality of surgical care. National VA Surgical Quality Improvement Program. Ann Surg 1998;228(4):491-507.

12. Ligush J, Jr., Pearce JD, Edwards MS, et al. Analysis of medical risk factors and outcomes in patients undergoing open versus endovascular abdominal aortic aneurysm repair. J Vasc Surg 2002;36(3):492-9.

13. Lee TH, Marcantonio ER, Mangione CM, et al. Derivation and prospective validation of a simple index for prediction of cardiac risk of major noncardiac surgery. Circulation 1999;100(10):1043-9. 
14. Birkmeyer JD, Finlayson EV, Birkmeyer CM. Volume standards for high-risk surgical procedures: potential benefits of the Leapfrog initiative. Surgery 2001;130(3):415-22.

15. Halm EA, Lee C, Chassin MR. Is volume related to outcome in health care? A systematic review and methodologic critique of the literature. Ann Intern Med 2002;137(6):511-20.

16. Dardik A, Lin JW, Gordon TA, Williams GM, Perler BA. Results of elective abdominal aortic aneurysm repair in the 1990s: A population-based analysis of 2335 cases. J Vasc Surg 1999;30(6):985-95.

17. Lemeshow S, Hosmer DW, Jr. A review of goodness of fit statistics for use in the development of logistic regression models. Am J Epidemiol 1982;115(1):92-106.

18. Harrell FE, Jr., Califf RM, Pryor DB, Lee KL, Rosati RA. Evaluating the yield of medical tests. Jama 1982;247(18):2543-6.

19. Hosmer DW, Taber S, Lemeshow S. The importance of assessing the fit of logistic regression models: a case study. Am J Public Health 1991;81(12):1630-5.

20. Rubin DB. Estimating causal effects from large data sets using propensity scores. Ann Intern Med 1997;127(8 Pt 2):757-63.

21. Becquemin J, Bourriez A, D'Audiffret A, et al. Mid-term results of endovascular versus open repair for abdominal aortic aneurysm in patients anatomically suitable for endovascular repair. Eur J Vasc Endovasc Surg 2000;19(6):656-61.

22. Thompson JP, Boyle JR, Thompson MM, Strupish J, Bell PR, Smith G. Cardiovascular and catecholamine responses during endovascular and conventional abdominal aortic aneurysm repair. Eur J Vasc Endovasc Surg 1999;17(4):326-33.

23. Salartash K, Sternbergh WC, 3rd, York JW, Money SR. Comparison of open transabdominal AAA repair with endovascular AAA repair in reduction of postoperative stress response. Ann Vasc Surg 2001;15(1):53-9.

24. Baxendale BR, Baker DM, Hutchinson A, Chuter TA, Wenham PW, Hopkinson BR. Haemodynamic and metabolic response to endovascular repair of infra-renal aortic aneurysms. Br J Anaesth 1996;77(5):581-5.

25. Kazis LE, Ren XS, Lee A, et al. Health status in VA patients: results from the Veterans Health Study. Am J Med Qual 1999;14(1):28-38.

26. Petersen LA, Normand SL, Daley J, McNeil BJ. Outcome of myocardial infarction in Veterans Health Administration patients as compared with medicare patients. $N$ Engl J Med 2000;343(26):1934-41.

27. Tassiopoulos AK, Kwon SS, Labropoulos N, et al. Predictors of early discharge following open abdominal aortic aneurysm repair. Ann Vasc Surg 2004;18(2):218-22.

28. Brown PM, Pattenden R, Vernooy C, Zelt DT, Gutelius JR. Selective management of abdominal aortic aneurysms in a prospective measurement program. J Vasc Surg 1996;23(2):213-20; discussion 221-2. 
29. Ashton CM, Souchek J, Petersen NJ, et al. Hospital use and survival among Veterans Affairs beneficiaries. N Engl J Med 2003;349(17):1637-46.

30. Elkouri S, Martelli E, Gloviczki P, et al. Most patients with abdominal aortic aneurysm are not suitable for endovascular repair using currently approved bifurcated stent-grafts. Vasc Endovascular Surg 2004;38(5):401-12.

31. Woodburn KR, Chant H, Davies JN, Blanshard KS, Travis SJ. Suitability for endovascular aneurysm repair in an unselected population. Br J Surg 2001;88(1):77-81.

32. Dimick JB, Pronovost PJ, Cowan JA, Ailawadi G, Upchurch GR, Jr. The volume-outcome effect for abdominal aortic surgery: differences in casemix or complications? Arch Surg 2002;137(7):828-32.

33. Birkmeyer JD, Siewers AE, Finlayson EV, et al. Hospital volume and surgical mortality in the United States. $N$ Engl J Med 2002;346(15):1128-37.

34. Scott RA, Ashton HA, Kay DN. Routine ultrasound screening in management of abdominal aortic aneurysm. $\mathrm{Br} \mathrm{Med} J$ (Clin Res Ed) 1988;296(6638):1709-10.

35. Fleming C, Whitlock EP, Beil TL, Lederle FA. Screening for abdominal aortic aneurysm: a best-evidence systematic review for the U.S. Preventive Services Task Force. Ann Intern Med 2005;142(3):203-11.

36. Ashton HA, Buxton MJ, Day NE, et al. The Multicentre Aneurysm Screening Study (MASS) into the effect of abdominal aortic aneurysm screening on mortality in men: a randomised controlled trial. Lancet 2002;360(9345):1531-9.

37. Norman PE, Jamrozik K, Lawrence-Brown MM, et al. Population based randomised controlled trial on impact of screening on mortality from abdominal aortic aneurysm. Bmj 2004;329(7477):1259.

38. Scott RA, Wilson NM, Ashton HA, Kay DN. Influence of screening on the incidence of ruptured abdominal aortic aneurysm: 5-year results of a randomized controlled study. Br J Surg 1995;82(8):1066-70.

39. Tisi PV, McLain AD, Jeddy TA, Ashton HA, Scott RA. Screening for abdominal aortic aneurysm: is opportunistic detection a realistic alternative? Eur J Vasc Endovasc Surg 1998;15(6):532-4.

40. Screening for abdominal aortic aneurysm: recommendation statement. Ann Intern Med 2005;142(3):198-202. 
Table 1. Demographics and baseline clinical characteristics of patients undergoing elective AAA repair.

\begin{tabular}{|c|c|c|c|}
\hline & \multicolumn{2}{|c|}{$\begin{array}{c}\text { Type of Surgery } \\
\text { N (\%) }\end{array}$} & \multirow[t]{2}{*}{$P$ value } \\
\hline & Open & EVAR & \\
\hline Total Patients & $1240(63.4)$ & $717(36.6)$ & NA \\
\hline $\begin{array}{l}\text { Demographic factors } \\
\text { Age (mean, (SD) yrs) }\end{array}$ & $70.2(7.89)$ & $71.6(7.78)$ & 0.0003 \\
\hline$<60$ & $131(10.6)$ & $50(7.0)$ & \multirow{3}{*}{0.0096} \\
\hline $60-79$ & $963(77.6)$ & $562(78.4)$ & \\
\hline$\geq 80$ & $146(11.8)$ & $105(14.6)$ & \\
\hline Male gender & $1228(99.0)$ & $714(99.6)$ & 0.1794 \\
\hline Race $\quad$ Non-Hispanic white & $995(80.2)$ & $573(80.0)$ & \multirow{4}{*}{0.7336} \\
\hline Black & $73(5.9)$ & $48(6.7)$ & \\
\hline Hispanic & $38(3.1)$ & $17(2.3)$ & \\
\hline Other/unspecified & $134(10.8)$ & $79(11.0)$ & \\
\hline \multicolumn{4}{|l|}{$\begin{array}{l}\text { Preoperative factors (NSQIP } \\
\text { comorbidities) }\end{array}$} \\
\hline ASA classification & & & \\
\hline High & $328(26.4)$ & $192(26.8)$ & \multirow{3}{*}{0.4081} \\
\hline Moderate & $859(69.3)$ & $503(70.1)$ & \\
\hline Low & $53(4.3)$ & $22(3.1)$ & \\
\hline
\end{tabular}




\begin{tabular}{|c|c|c|c|}
\hline History of COPD & $320(25.8)$ & $191(26.6)$ & 0.6863 \\
\hline History of $\mathrm{CHF}$ & $32(2.6)$ & $26(3.6)$ & 0.1888 \\
\hline Hepatic insufficiency & $41(3.3)$ & $22(3.1)$ & 0.7737 \\
\hline Cerebrovascular disease/neuro & $320(25.8)$ & $152(21.2)$ & 0.0217 \\
\hline Malignancy & $13(1.1)$ & $8(1.1)$ & 0.8892 \\
\hline \multicolumn{4}{|l|}{ Diabetes mellitus } \\
\hline Insulin-dependent & $38(3.1)$ & $33(4.6)$ & \multirow{3}{*}{0.2120} \\
\hline NIDDM & $126(10.2)$ & $70(9.8)$ & \\
\hline No diabetes & $1076(86.8)$ & 614 & \\
\hline Chronic renal disease & $13(1.1)$ & $8(1.1)$ & 0.8892 \\
\hline $\begin{array}{l}\text { Smoking history (within last } \\
\text { year) }\end{array}$ & $568(45.8)$ & $283(39.5)$ & 0.0064 \\
\hline \multicolumn{4}{|l|}{ Functional status } \\
\hline Partially dependent & $69(5.6)$ & $43(6.0)$ & \multirow{3}{*}{0.7644} \\
\hline Totally dependent & $6(0.5)$ & $5(0.7)$ & \\
\hline Independent & $1834(93.7)$ & $669(93.3)$ & \\
\hline Serum creatinine $\geq 2.0 \mathrm{mg} / \mathrm{dL}$ & $81(6.5)$ & $36(5.0)$ & 0.1742 \\
\hline \multicolumn{4}{|l|}{$\begin{array}{l}\text { Preoperative factors } \\
\text { (PTF and OPC } \\
\text { comorbidities) }\end{array}$} \\
\hline Angina & $114(9.2)$ & $67(9.3)$ & 0.9116 \\
\hline Angina-unstable & $41(3.3)$ & $33(4.6)$ & 0.1475 \\
\hline
\end{tabular}




\begin{tabular}{|l|c|c|c|}
\hline Angina-both & $140(11.3)$ & $89(12.4)$ & 0.4567 \\
\hline MI (remote) & $669(54.0)$ & $381(53.1)$ & 0.7280 \\
\hline CABG & $163(13.2)$ & $102(14.2)$ & 0.5008 \\
\hline PTCA & $40(3.2)$ & $32(4.5)$ & 0.1613 \\
\hline Arrhythmia & $277(22.3)$ & $150(20.9)$ & 0.4642 \\
\hline Cardiovascular disease & $58(4.7)$ & $24(3.4)$ & 0.1571 \\
\hline Ischemic heart disease & $47(3.8)$ & $34(4.7)$ & 0.3085 \\
\hline Hypertension & $936(75.5)$ & $532(74.2)$ & 0.5267 \\
\hline Hyperlipidemia & $661(53.3)$ & $380(53.0)$ & 0.8954 \\
\hline COPD & $427(34.4)$ & $223(31.1)$ & 0.1314 \\
\hline CHF & $150(12.1)$ & $103(14.4)$ & 0.1495 \\
\hline CRI, ESRD & $78(6.3)$ & $38(5.3)$ & 0.3713 \\
\hline Hypertensive renal failure & $39(3.2)$ & $18(2.5)$ & 0.4211 \\
\hline Renal Insufficiency & $98(7.9)$ & $57(8.0)$ & 0.9707 \\
\hline Cirrhosis & $7(0.6)$ & $12(1.7)$ & 0.0159 \\
\hline Stroke history & $31(2.5)$ & $18(2.5)$ & 0.9886 \\
\hline Atherosclerotic carotid lesion & $29(2.3)$ & $8(1.1)$ & 0.0556 \\
\hline TIA & $31(2.5)$ & $12(1.7)$ & 0.2296 \\
\hline CVA & $49(4.0)$ & $22(3.1)$ & 0.3140 \\
\hline Carotid lesion & $248(20.0)$ & $113(15.8)$ & 0.0198 \\
\hline Low hospital Volume & & $114(15.9)$ & $<0.001$ \\
\hline & $392(31.6)$ & & \\
\hline & & & \\
\hline & & & \\
\hline
\end{tabular}


Definitions: ASA, American Society of Anesthesiology; COPD, chronic obstructive pulmonary disease; CHF, congestive heart failure; IDDM, insulin dependent diabetes mellitus; $\mathrm{MI}$, myocardial infarction; CABG, coronary artery bypass graft; PTCA, percutaneous transluminal coronary angioplasty; CRI, chronic renal insufficiency; ESRD, end stage renal disease; RF, renal failure; TIA, transient ischemic attack; CVA, cerebrovascular accident 
Table 2. Unadjusted outcome rates following open repair and EVAR.

\begin{tabular}{|l|c|c|c|}
\hline & \multicolumn{2}{|c|}{ Type of Surgery } & \\
\hline Mortality and & Open & EVAR & P value \\
$\mathbf{n}(\%)$ & & & \\
\hline 30-day mortality & $71(5.7)$ & $22(3.1)$ & 0.0079 \\
\hline 365 -day mortality & $149(12.0)$ & $60(8.4)$ & 0.0121 \\
\hline Any complication ${ }^{1}$ & $362(29.2)$ & $114(15.9)$ & $<0.0001$ \\
\hline Cardiac morbidity & $69(5.6)$ & $14(1.9)$ & $<0.0001$ \\
\hline $\begin{array}{l}\text { Neurological morbidity } \\
\text { Pulmonary }\end{array}$ & $10(0.51)$ & $8(0.4)$ & 0.4952 \\
\hline Renal dysfunction & $48(2.5)$ & $16(0.8)$ & 0.0433 \\
\hline Wound complication & $73(3.7)$ & $28(1.4)$ & 0.0515 \\
\hline Graft failure & $7(0.36)$ & $15(0.77)$ & 0.002 \\
\hline days) & $10.9 \pm 14.3$ & $4.6 \pm 7.2$ & $<0.0001$ \\
\hline (mean \pm SD; days) & & & $<0.0001$ \\
\hline ICU stay (mean \pm SD; & $6.3 \pm 11.6$ & $2.8 \pm 8.6$ & \\
\hline & & & \\
\hline & & & \\
\hline & & & \\
\hline & & & \\
\hline
\end{tabular}

${ }^{1}$ Includes all 21 NSQIP postoperative complication variables.

Definitions: LOS, length of stay; ICU, Intensive care unit. 
Table 3. Adjusted and unadjusted odds ratios following EVAR

\begin{tabular}{|c|c|c|c|c|c|c|}
\hline & \multicolumn{3}{|c|}{ Adjusted } & \multicolumn{3}{|c|}{ Unadjusted } \\
\hline & OR & $95 \% \mathrm{CI}$ & $\begin{array}{c}\text { P } \\
\text { value }\end{array}$ & OR & $95 \% \mathrm{CI}$ & $\begin{array}{c}\mathbf{P} \\
\text { value }\end{array}$ \\
\hline $\begin{array}{l}\text { 30-day } \\
\text { mortality }\end{array}$ & 0.58 & $0.35,0.97$ & 0.0367 & 0.52 & $0.32,0.85$ & 0.0079 \\
\hline $\begin{array}{l}\text { 365-day } \\
\text { mortality }\end{array}$ & 0.59 & $0.42,0.84$ & 0.0028 & 0.67 & $0.49,0.92$ & 0.0121 \\
\hline
\end{tabular}


Table 4. Unadjusted Odds Ratios of Complications Resulting from EVAR

Compared to Open Repair.

\begin{tabular}{|c|c|c|c|}
\hline & $\begin{array}{l}\text { Odds } \\
\text { Ratio }\end{array}$ & $\begin{array}{c}95 \% \text { Confidence } \\
\text { Interval }\end{array}$ & P value \\
\hline Any complication $^{1}$ & 0.46 & $0.36,0.58$ & $<0.0001$ \\
\hline \multicolumn{4}{|l|}{ Specific Categories } \\
\hline Cardiac & 0.34 & $0.19,0.60$ & 0.0001 \\
\hline Neurological & 1.39 & $0.54,3.53$ & 0.4898 \\
\hline Pulmonary & 0.35 & $0.24,0.51$ & $<0.0001$ \\
\hline Renal dysfunction & 0.57 & $0.43,2.58$ & 0.8892 \\
\hline Wound & 0.65 & $0.42,1.01$ & 0.056 \\
\hline Graft failure & 3.76 & $1.53,9.28$ & 0.002 \\
\hline
\end{tabular}

'Any complication denotes unadjusted rates, which include all 21 NSQIP postoperative complication categories. 
Table 5. Summary of recommendations for screening for AAA by the U.S. Preventative Services Task Force.

\begin{tabular}{|l|l|}
\hline Rating & Recommendation \\
\hline B & $\begin{array}{l}\text { One-time screening for AAA by ultrasonography in men aged } 65-75 \\
\text { who have ever smoked }\end{array}$ \\
\hline C & $\begin{array}{l}\text { No recommendation for or against screening for AAA in men aged 65- } \\
75 \text { who have never smoked }\end{array}$ \\
\hline D & Recommends against routing screening for AAA in women \\
\hline
\end{tabular}


Figure 1.a 30-day survival.

Kaplan-Meier Survival Curve for Time to Death by Surgery Group

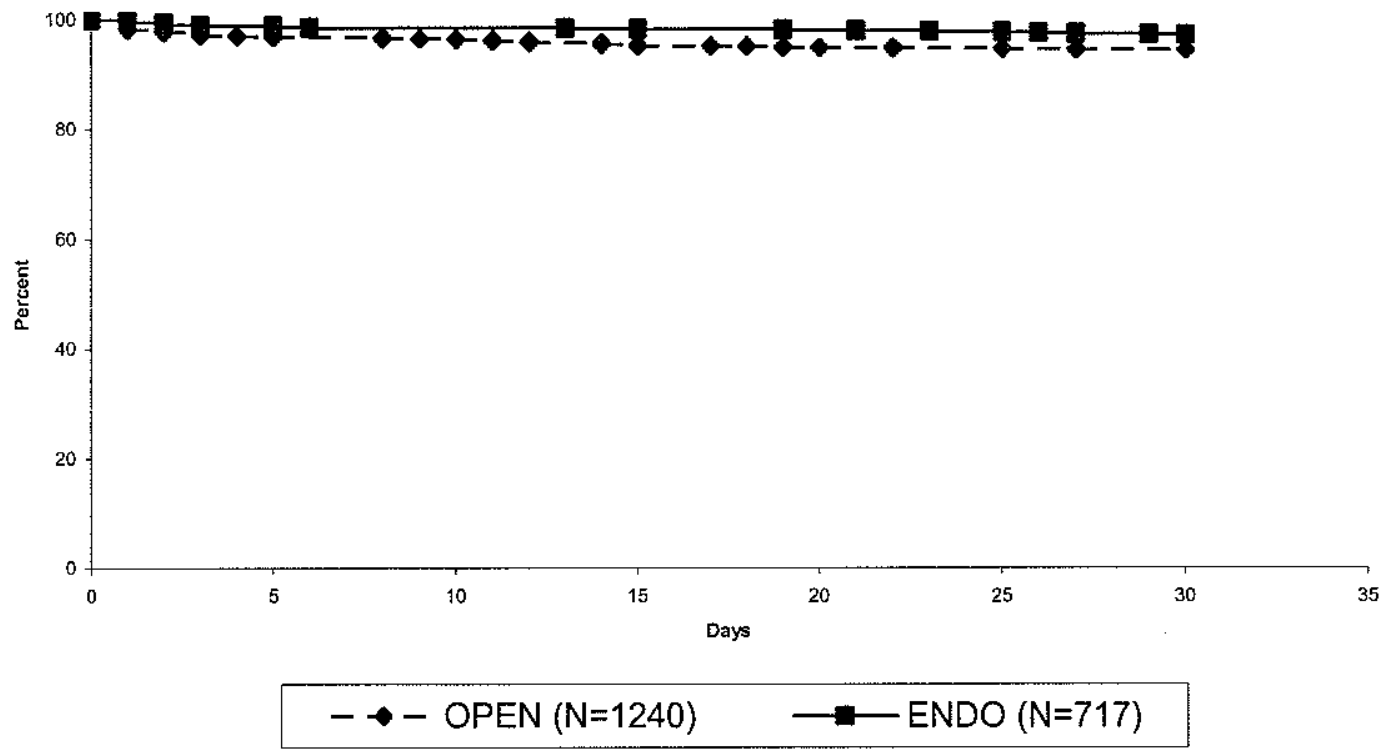


34

Figure 1.b 1-year survival curve

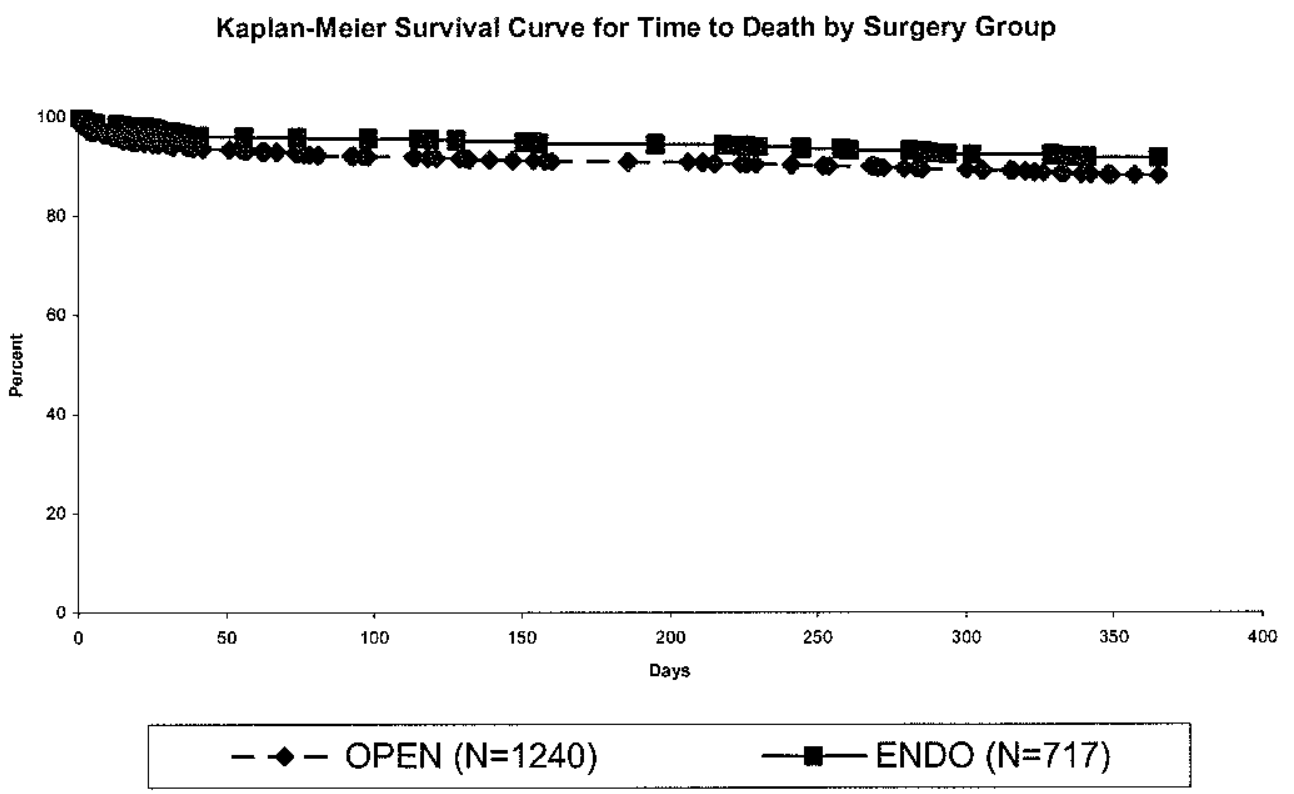


Figure 2. Propensity score curves for EVAR versus open repair.
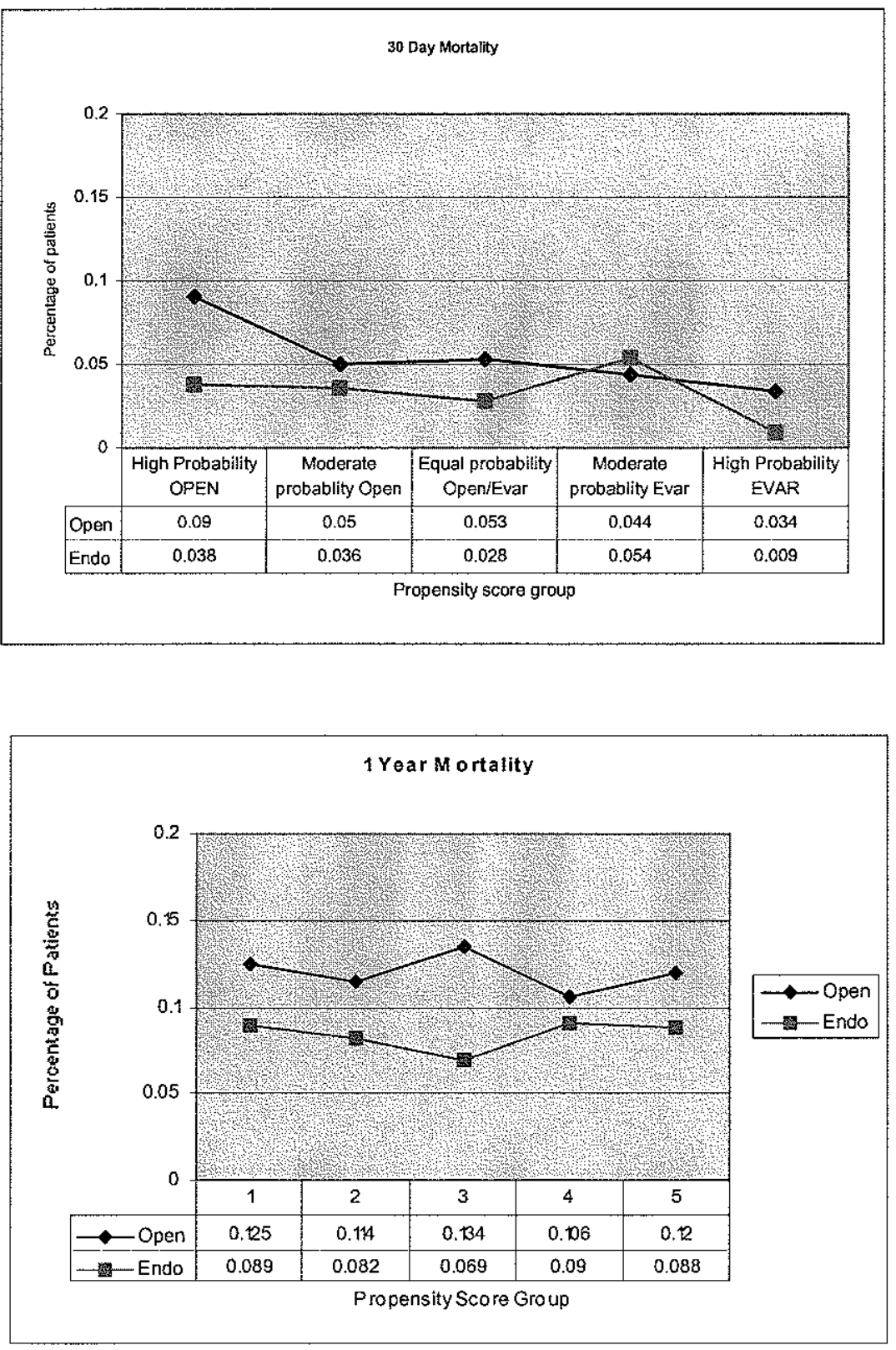